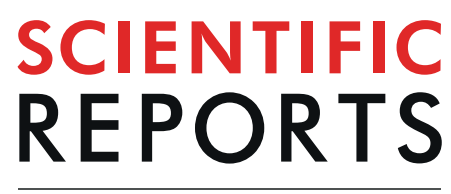

natureresearch

Check for updates

\title{
The role of early social rearing, neurological, and genetic factors on individual differences in mutual eye gaze among captive chimpanzees
}

\author{
William D. Hopkins ${ }^{1 凶}$, Michele M. Mulholland $\mathbb{1}^{1,2 \bowtie}$, Lisa A. Reamer ${ }^{1}$,
} Mary Catherine Mareno ${ }^{1}$ \& Steven J. Schapiro ${ }^{1,3}$

\begin{abstract}
Mutual eye gaze plays an important role in primate social development and communication. In the current study, we examined the underlying experiential, genetic, and neuroanatomical basis of mutual eye gaze variation in adult captive chimpanzees. A multivariate analysis of variance revealed a significant rearing effect on bout length, with human-reared chimpanzees engaging in longer bouts of mutual gaze compared to mother-reared and wild-born individuals. Next, we utilized source-based morphometry (SBM) to examine gray matter covariation in magnetic resonance imaging scans and determine the relationship between the resulting gray matter covariation components and mutual eye gaze. One SBM component was negatively correlated with gaze duration (nucleus accumbens and anterior insular cortex), while two components were positively correlated with bout length (posterior cingulate cortex, inferior occipital cortex, middle temporal cortex, hippocampus, and the precentral sulcus). Finally, heritability analyses revealed mutual eye gaze to be modestly heritable and significant genetic correlations between bout length and two gray matter covariation components. This study reveals that non-genetic factors, and to a lesser extent, genetic factors appear to influence mutual eye gaze in adult chimpanzees, and is the first to report neuroanatomical correlates of mutual eye gaze variation in chimpanzees.
\end{abstract}

Visual communication through mutual eye gaze and gaze following play important roles in social development and nonverbal communication in human and nonhuman primates ${ }^{1-5}$. For instance, early in life, infants and their caregivers engage in mutual eye gaze and many have suggested that this early nonverbal communication has a facilitating effect in the formation of cognition, social bonding, and attachment ${ }^{6-8}$. Further, deficits in mutual eye gaze have been reported in clinical populations that are characterized as exhibiting atypical social, behavioral, and socio-communicative skills, such as schizophrenia and autism spectrum disorder ${ }^{9-11}$. Others have suggested that mutual eye gaze plays a role in the ways that individuals make inferences about what others can see and may think. Indeed, Grossmann ${ }^{1}$ hypothesized that, in humans, responses to mutual eye gaze and gaze following play the key functions of detecting the presence, inferring the content, and fostering collaboration with other minds.

Given the importance of visual communication in primate communicative signaling, researchers have published many comparative studies on the structure of the eye, as well as the function of gaze in communicative behavior in nonhuman primate taxa, from prosimians to great apes ${ }^{12-20}$. The collective findings suggest that information conveyed through mutual eye gaze and gaze following is widespread among primate species, and therefore, has a strong evolutionary foundation. However, our understanding of individual and phylogenetic variation in the neural and biological foundations of mutual eye gaze and gaze following is limited and relatively understudied $^{9,16,21-24}$.

In the current study, one goal was to examine the independent and potential interactive effect of experiential and genetic factors on chimpanzee mutual eye gaze (MEG). Our motivation for this aspect of the study was

${ }^{1}$ Department of Comparative Medicine, The University of Texas MD Anderson Cancer Center, Michale E. Keeling Center for Comparative Medicine and Research, 650 Cool Water Drive, Bastrop, Texas, 78602, USA. ${ }^{2}$ Center for Behavioral Neuroscience, Georgia State University, 100 Piedmont Ave SE, Atlanta, Georgia, 30303, USA. ${ }^{3}$ Department of Experimental Medicine, University of Copenhagen, Blegdamsvej 3, 2200 Copenhagen N, Copenhagen, Denmark. ${ }^{凶}$-mail:wdhopkins@mdanderson.org; mmmulholland@mdanderson.org 


\begin{tabular}{|l|l|l|l|}
\hline Frequency & Trial 2 & Trial 3 & Trial 4 \\
\hline & 0.354 & 0.283 & 0.368 \\
\hline Trial 1 & - & 0.417 & 0.344 \\
\hline Trial 2 & - & 0.403 & \\
\hline Trial 3 & \multicolumn{5}{l|}{} \\
\hline Duration & Trial 2 & Trial 3 & Trial 4 \\
\hline \multicolumn{5}{|l|}{0.409} & 0.394 & 0.395 \\
\hline Trial 1 & - & 0.454 & 0.552 \\
\hline Trial 2 & - & 0.542 & \\
\hline Trial 3 & - & Trial 3 & Trial 4 \\
\hline Bout Length & Trial 2 & 0.351 & 0.290 \\
\hline \multicolumn{5}{|l|}{} \\
\hline Trial 1 & 0.303 & 0.366 & 0.576 \\
\hline Trial 2 & - & 0.428 & \\
\hline Trial 3 & - &
\end{tabular}

Table 1. Pearson Product-Moment Correlation Coefficients between Mutual Eye Gaze Frequency, Duration and Bout Length Across the 4 Trials. All correlation coefficients were significant at $p<0.001$.

two-fold. First, though there is widespread comparative interest in gaze-related behavior, such as mutual eye gaze and gaze following in nonhuman primates, there are few studies that have examined the potential role of genetic factors underlying individual variation in relatively large cohorts of individuals. Consistent with previous studies in nonhuman primates that utilize pedigree information from captive populations, we used the software program SOLAR to estimate heritability in the MEG measures ${ }^{25-32}$. Given the strong evolutionary foundations for MEG observed in primates, we hypothesized that MEG would be significantly heritable.

Second, in addition to estimating the contribution of genetic factors to MEG, we also sought to examine how experiential factors may contribute to individual variation in MEG. Specifically, in chimpanzees and other great apes, there is some evidence that early rearing experiences have a significant influence on measures of social cognition, including gaze following, and in responses to socio-communicative cues, such as gazing and manual pointing. In the majority of these studies, chimpanzees with extensive experience with humans, or enculturated apes as they are sometimes called, outperform apes raised in standard laboratory settings ${ }^{20,33-37}$. One interpretation of the enhanced performance of encultured apes is that they are more sensitive to or have learned to better respond to human socio-communicative cues than chimpanzees raised by, and with, their conspecifics. Because humans often serve as the experimenter in studies of social cognition in apes, the suggestion is that they perform better because they are better able to utilize early experiences with humans on certain tasks. To examine how different experiences may influence MEG, we evaluated the role of early social rearing on MEG variation by comparing chimpanzees reared by humans, to those raised by their biological mothers in captivity, or were born in the wild. Because the human-reared apes in the current study were more familiar with humans early in life, it was hypothesized that these chimpanzees would engage in mutual eye gaze more frequently, and for longer durations, than conspecific mother-reared or wild-born apes after controlling for their genetic relatedness.

A second goal of this study was to examine the neuroanatomical correlates of MEG in chimpanzees. MEG measures were correlated with gray matter covariation component scores derived from a source-based morphometry (SBM) analysis ${ }^{38}$ performed on magnetic resonance image scans obtained in the chimpanzee sample. Our research group has previously performed SBM analysis on scans from a sample of captive chimpanzees (the majority of which were subjects in the current study) and found between 19 and 24 spatial components, depending on the preprocessing methods ${ }^{25}$. Here, phenotypic measures of MEG were correlated with the weighted scores derived from each component to determine whether individual variation was associated with covariation scores for any of the components. Finally, to test whether any phenotypic associations we found between the MEG scores and the SBM components had a potential common genetic basis, we used quantitative genetics to test for genetic correlations in our chimpanzee sample.

\section{Results}

Reliability and the effect of sex and rearing on mutual eye gaze. Pearson's product-moment correlations were performed to test for consistency in MEG responses across the four trials and these findings are shown in Table 1. We found significant positive associations among the four trials for mutual eye gaze frequency, duration, and bout length. Thus, the chimpanzee responses were consistent and repeatable across test trials. For this reason, we averaged the frequency, duration, and bout length measures of mutual eye gaze across all four trials and these three variables served as the outcome measures for all subsequent analyses. We also assessed inter-observer reliability by having two researchers collect simultaneous data, with their heads side-by-side, from a subset of chimpanzees $(\mathrm{NCCC}=40$; YNPRC $=11$ ). This is by no means a perfect assessment of reliability, but both frequency $(r=0.89-0.92)$ and duration $(r=0.93-0.96)$ were highly positively correlated between observers.

We next considered the effect of sex and rearing on each average mutual eye gaze outcome measure. For this analysis, a multivariate analysis of covariance was initially performed, with the mean frequency, duration, and bout length in mutual eye gaze serving as the dependent measures, age as a covariate, and sex and rearing history as the fixed factors. An overall significant main effect for rearing was found $[F(6,422)=2.595, p=0.018]$. The 


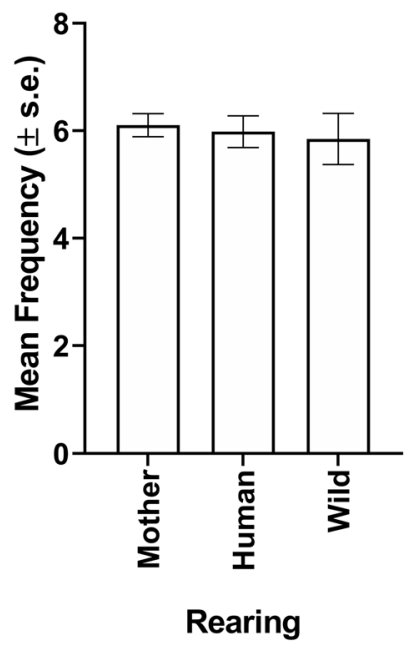

(a)

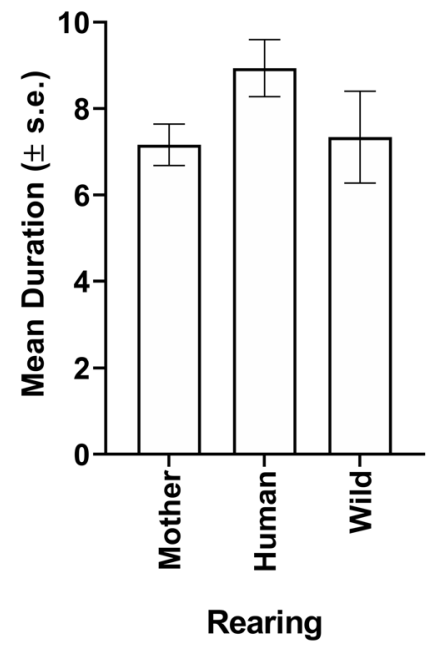

(b)

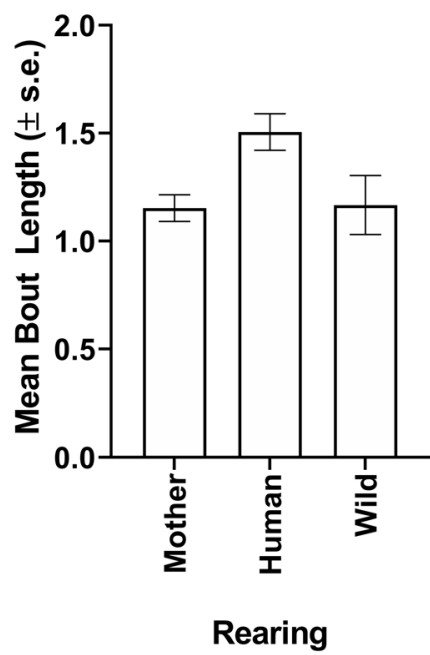

(c)

Figure 1. Mean and standard errors bars for mutual eye gaze (a) frequency (b) duration and (c) bout length in the mother-reared, human-reared, and wild-born chimpanzees.

subsequent univariate $F$-tests revealed significant rearing effects on bout length $[F(2,211)=6.5 f 15, p=0.002]$, and a borderline effect for duration $[F(2,211)=2.689, p=0.070]$. There was no significant effect for frequency $[F(2,211)=0.124, p=0.883]$. The mean mutual eye gaze frequency, duration, and bout length are shown in Fig. 1. For bout length and duration, post-hoc analyses using Tukey's Honestly Significant Difference tests, indicated that human-reared chimpanzees had longer bout durations than mother-reared and wild-born individuals, but these latter two groups did not differ significantly from each other. Human-reared chimpanzees had longer total durations than both mother-reared and wild-born chimpanzees, but this difference was only significant for wild-born individuals. There was no significant difference in mutual eye gaze across the three rearing conditions.

Phenotypic associations between mutual eye gaze and the SBM correlates of mutual eye gaze. We next tested for associations between the different measures of mutual eye gaze and the weighted score for each SBM component. Partial correlation coefficients were performed between the mutual eye gaze frequency, duration, and bout length measures and the subject's weighted score for each of the 18 SBM components, with scanner magnet, sex, age, and rearing history as covariates. We found no significant associations between the frequency in mutual eye gaze and any of the SBM components. For the duration in mutual eye gaze, a significant positive association was found with SBM component $7(r=0.163, p=0.026)$ and a negative association with SBM component 18 ( $r=-0.145, p=0.049)$. Lastly, for mutual eye gaze bout length, significant positive associations were found with SBM components 7 and $10(r=0.174, p=0.018$ and $r=0.212, p=0.004$, respectively), and a significant negative association with component $18(r=-0.160, p=0.030)$. Scatterplots showing the association between mutual eye gaze duration and bout length, and the weighted scores for SBM components 7, 10 and 18 are shown in Figs. 2 through 4. SBM component 7 was comprised of the posterior cingulate cortex (bilateral) and inferior occipital cortex (bilateral). SBM component 10 was made up of bilateral anterior and posterior middle temporal cortex, tail of the hippocampus and the ventral portion of the left inferior precentral sulcus. SBM component 18 was comprised of two main regions, including the inferior basal forebrain, which included the nucleus accumbens and anterior insular cortex.

Heritability and genetic correlations between mutual eye gaze and SBM components. In the next set of analyses, we estimated heritability for the measures of MEG and those SBM components (7, 10 and 18) that were found to be phenotypically associated with them. Table 2 shows the heritability estimates and contributions of the covariates on each MEG phenotype and SBM components 7, 10, and 18. As can be seen, significant heritability was found for frequency in mutual eye gaze, while borderline significant heritability was found for duration and mean bout length. With respect to the SBM components, all three were significantly heritable. Not surprisingly, scanner magnet also accounted for a significant proportion of variance, as did to a lesser extent, sex and age. We next tested for genetic correlations between the mutual eye gaze mean bout length data and the weighted scores for SBM components 7, 10 and 18, respectively. We found a significant genetic correlation between mutual eye gaze mean bout length and SBM component 7 (rhoG $=0.896, \mathrm{SE}=0.707, \mathrm{p}=0.007)$ and a borderline significant correlation with component $10(\mathrm{rhoG}=0.872, \mathrm{SE}=1.14, \mathrm{p}=0.073)$. There was no significant genetic correlation between mutual eye gaze bout length and SBM component 18 .

\section{Discussion}

There were five main findings in the current study. First, we found consistent MEG responding across four test trials separated by at least one day. Thus, as a measure of individual variation, MEG seems to be a reliable measure. Second, we found that early social rearing experiences had a significant impact on mutual eye gaze bout length. Third, measures of MEG were modestly heritable in our sample of chimpanzees. Fourth, we found significant 
(a)
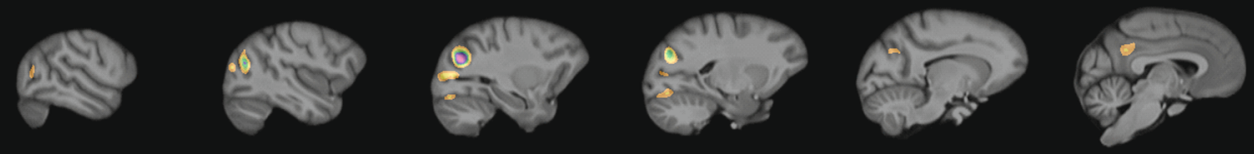

(b)
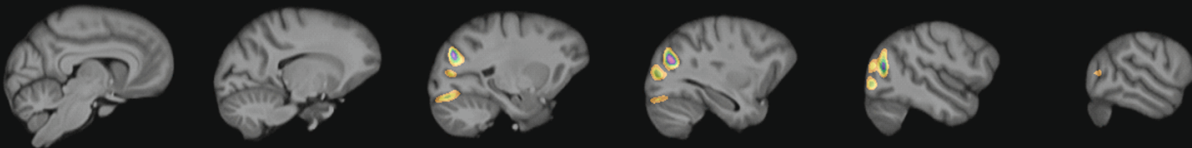

(c)
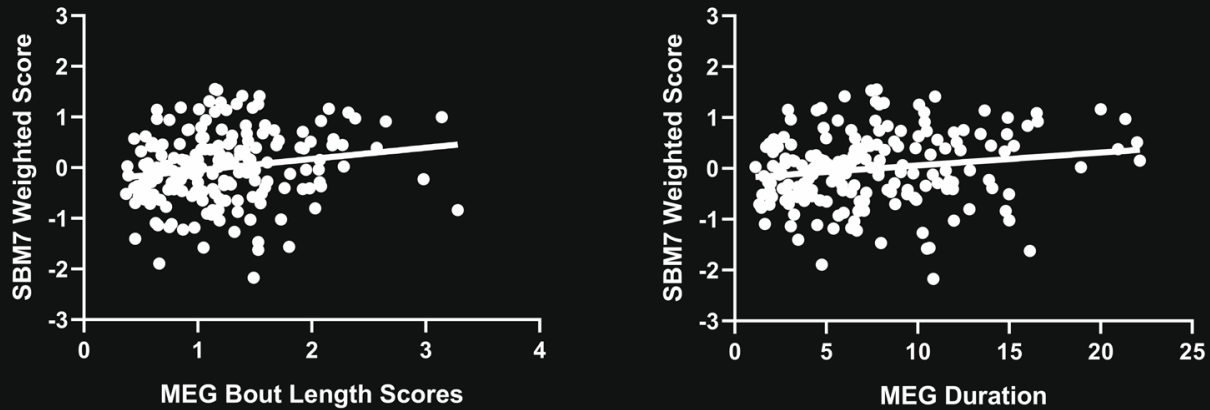

Figure 2. (a,b) Serial sagittal views of visual cortical and the posterior cingulate regions that comprise SBM component 7, and (c) scatterplots of the association between SBM7 scores and mean bout length and overall duration. Note: Weighted scores are adjusted for covariates.

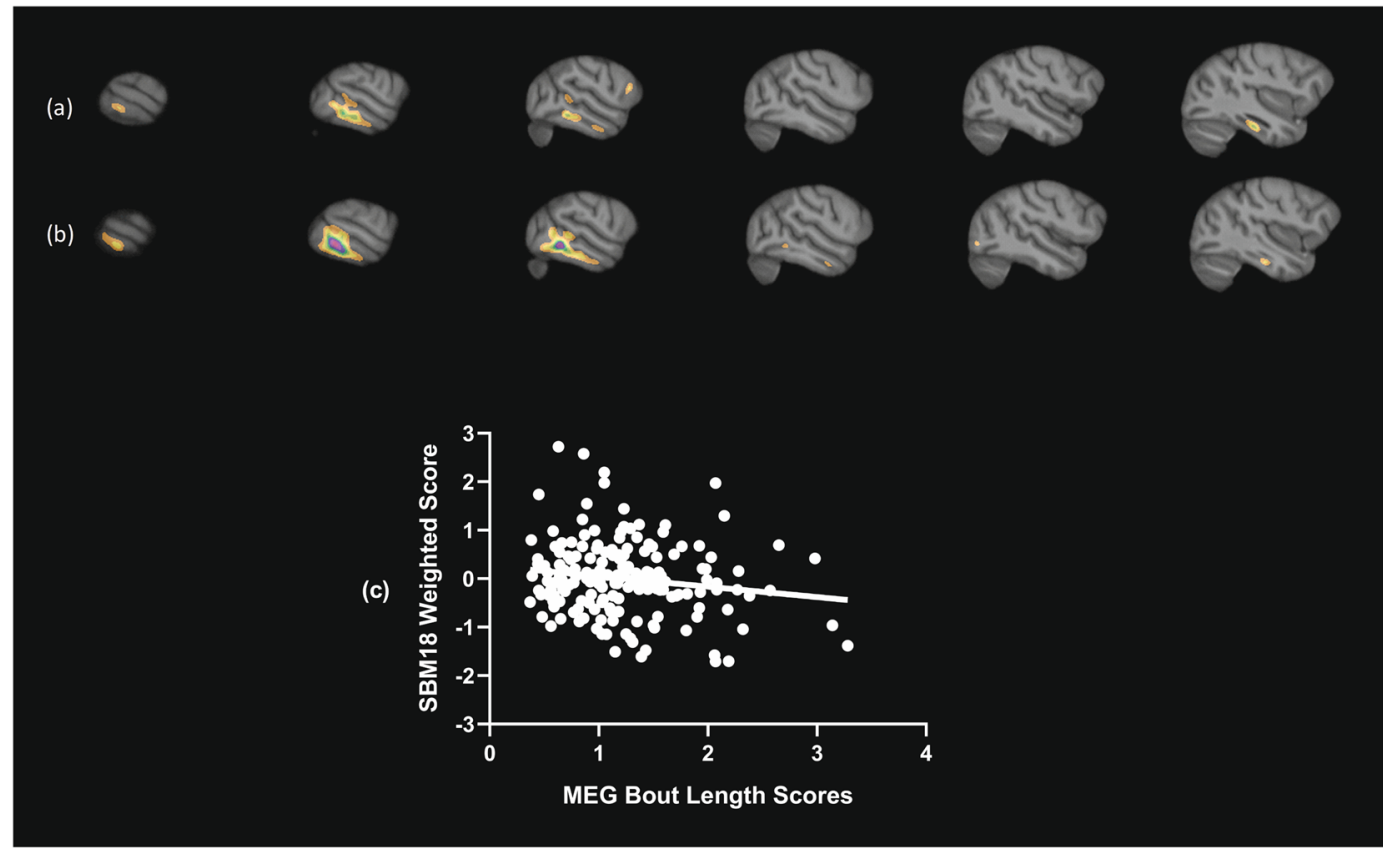

Figure 3. (a,b) Serial sagittal views of middle and inferior temporal lobe and hippocampal regions that comprise SBM component 10, and (c) scatterplot of the association between mean bout length and SBM10 scores. Note: Weighted scores are adjusted for covariates.

neuroanatomical associations with individual variation in MEG bout length and duration, but not frequency. Finally, we found significant genetic correlations between MEG bout length and two of the three gray matter covariation components. 


\begin{tabular}{|l|l|l|l|l|l|}
\hline Phenotype & $\mathbf{h}^{2}$ & s.e. & p & Covariates & Variance \\
\hline Mutual Gaze & 0.381 & 0.167 & 0.006 & Rearing & 0.034 \\
\hline Frequency & 0.183 & 0.148 & 0.081 & None & \\
\hline Duration & 0.186 & 0.129 & 0.050 & None & \\
\hline Bout Length & \multicolumn{7}{|l|}{} \\
\hline SBM Components & 0.765 & 0.134 & 0.000001 & Sex, Age, Scanner & 0.344 \\
\hline 7 & 0.907 & 0.109 & 0.000001 & Sex, Age, Scanner & 0.192 \\
\hline 10 & 0.391 & 0.146 & 0.001 & Age, Scanner & 0.312 \\
\hline 18 &
\end{tabular}

Table 2. Heritability of MEG Measures and SBM components.

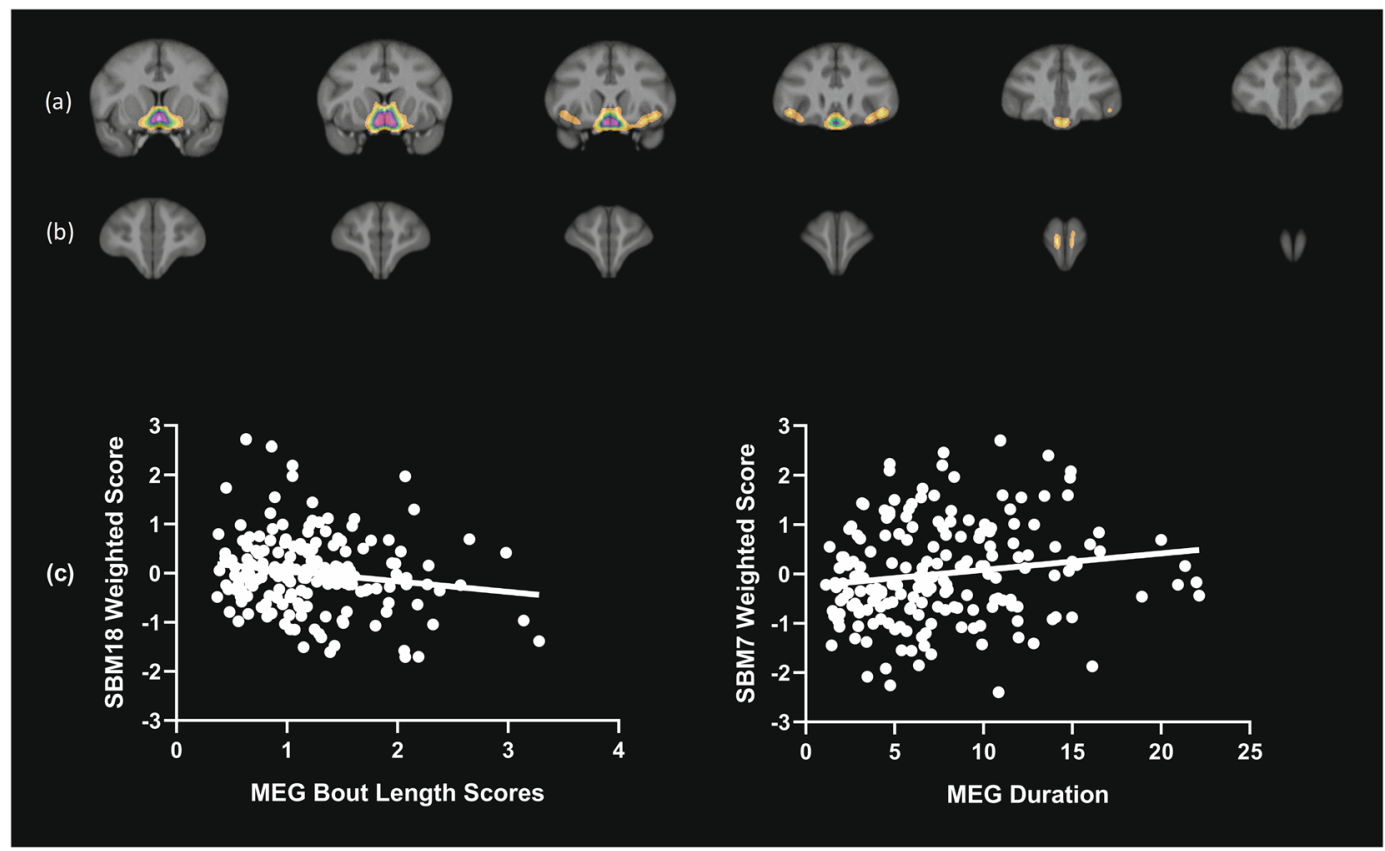

Figure 4. (a,b) Serial coronal views of basal forebrain, anterior insula and frontal pole regions that comprise SBM component 18, and (c) scatterplots of the association between SBM18 scores, and mean bout length and overall duration. Note: Weighted scores are adjusted for covariates.

Regarding rearing history, we found that chimpanzees reared by humans in a nursery setting engaged in mutual eye gaze for longer bout lengths compared to mother-reared and wild-born chimpanzees. There was also a borderline, but non-significant, effect of overall duration, with nursery-reared chimpanzees having longer overall duration of mutual eye gaze. There was no difference, however, in the frequency of mutual eye gaze across these rearing histories. These findings were consistent with our hypothesis, and suggest that early social rearing experiences can shape MEG and, perhaps, tune them toward social agents early in life as has been reported in humans ${ }^{39}$. It might be argued that the nursery-reared chimpanzees simply had more exposure to humans than mother-reared or wild-born apes, and this explains the results; however, the wild-born apes were older individuals that were brought to captivity before 1974, when the importation of wild chimpanzees ended. Thus, these apes have spent as much time around, and interacting with, humans as many, if not all, of the nursery-reared apes. If it was simply a matter of exposure to humans accounting for the rearing effect, then the data from the wild-born apes should be more similar to the nursery- than mother-reared apes. We believe what accounts for the increased duration and bout length in the nursery-reared chimpanzees is that they received this eye contact early in life facilitating their MEG responding as adults.

The findings showing increased MEG duration and bout length in nursery-reared compared to mother-reared chimpanzees are relevant to other existing literatures in, at least, two important ways. First, previous studies have shown that nursery-reared chimpanzees, particularly those with intensive interactions with humans, perform significantly better (or more similar to humans) on tasks that measure dimensions of social cognition, such as gaze following, joint attention, understanding of intentions, and the use of attention-getting behaviors compared to mother-reared apes ${ }^{33,34,40}$. In light of the fact that many of these cognitive tasks involve the chimpanzees monitoring of the experimenter's (a human) visual attention, it seems reasonable to suggest that early exposure to human mutual eye gaze may account for performance on these, and other, social cognition tasks. Second, there is some 
evidence that nursery-reared chimpanzees have a difficult time integrating in social groups of chimpanzees as juveniles, adolescents or adults, particularly if the existing group is comprised of a disproportionately large number of mother-reared individuals ${ }^{41-43}$. One potential explanation for the difficulty of nursery-reared chimpanzees to integrate into larger chimpanzee social groups comprised of mother-reared individuals is that they have not learned the appropriate species-specific functional uses of, and responses to, different aspects of mutual eye gaze in social contexts. This is not to suggest that they cannot or do not eventually learn them, but their acquisition of these skills is delayed and programmed differently, and therefore, requires additional experiences.

One limitation of this study was the fact we used a human experimenter to study MEG rather than trying to quantify these measures during intraspecific interactions between chimpanzees ${ }^{44}$. Thus, the ecological validity of these findings might be called into question. Measuring mutual eye gaze with a human observer, while likely less representative of natural gaze behaviors in the wild, is reliable and certainly more ecologically valid than using video or pictorial stimuli in studies involving eye tracking and related methodologies. Eye gaze behaviors and responses to faces are of great interest in both human and nonhuman primate research, most of which involves automated presentation of two-dimensional stimuli (videos or pictures) ${ }^{19,45-50}$. While eye tracking and related studies clearly show that features of the face are a focal point of interest, it is not necessarily the case that nonhuman primates view them as representational social stimuli ${ }^{51}$. Indeed, Rossion and Taubert ${ }^{48}$ have suggested that nonhuman primates do not see images on the screen as social stimuli, even though they can discriminate between faces and non-faces using these methods. In short, we believe that the approach used in this study is ecologically valid, though this could be improved with additional studies. While more difficult to collect, the frequency and duration of mutual eye gaze with a conspecific should be directly compared to those with a human observer.

MEG also showed moderate to low heritability, suggesting that genetic factors likely play only a small role in explaining individual variation in chimpanzees, a finding that did not support our hypothesis. We were somewhat surprised by this result, given (1) the role mutual eye gaze and gaze following play in social communication in chimpanzees and other primates and (2) our previous findings demonstrating heritability in gaze following and related social cognitive processes in chimpanzees ${ }^{52,53}$. One possibility is that the variability in MEG responses, particularly the average bout length, was relatively low, constraining our ability to detect stronger heritability (i.e., the range of responses was too small). Indeed, in humans, bonobos, chimpanzees, and monkeys, eye tracking studies of conspecific faces clearly show that the eyes are a region of interest (though there are species differences in amount of time spent looking at the eyes versus other regions) ${ }^{19,50}$. Thus, the eyes are a very potent visual stimulus in primates and automatic responses to facial stimuli likely attenuate individual variation in MEG. Alternatively, it may be that social learning and experiential factors play a far greater role in shaping the function and use of mutual eye gaze in social communication in primates than genetic factors, an interpretation partially supported by the rearing effects reported in this study.

We also found phenotypic associations between MEG bout length and three SBM components (7, 10 and 18) and genetic correlations for two components (7 and 10). Recall that SBM components 7 and 10 were comprised of visual cortex and portions of the inferior and middle temporal cortex. The regions within SBM components 7 and 10 are part of the visual processing stream, particularly the ventral pathway, and therefore, it is not surprising that these areas are both phenotypically and genetically associated with MEG. The association between SBM component 18 and MEG bout length is more difficult to explain, yet interesting for at least two reasons. First, it has been previously reported that mother- and nursery-reared chimpanzees differ on gray matter covariation in the basal forebrain ${ }^{54}$, and this region entirely overlaps with those described for component 18 in this study. Recall that nursery- and mother-reared chimpanzees differed in their MEG bout length, and that MEG bout length is associated with covariation in the basal forebrain region, suggesting that rearing experiences may mediate the association between MEG and gray matter covariation within the basal forebrain. Second, regions within the basal forebrain, including the caudate and nucleus accumbens, are rich in dopamine neurons, and some have suggested that they play an important role in the reward circuit of the brain ${ }^{55}$. It has also been suggested that mutual eye gaze activates oxytocin release during social interactions, which in turn, stimulates the release of dopamine ${ }^{56-58}$. This results in a feedback loop between oxytocin and dopamine that facilitates the reward value of mutual eye gaze during social interactions ${ }^{59}$. Therefore, the association between MEG bout length and SBM component 18 reported here, may reflect individual variation in the reward value of mutual eye gaze for the chimpanzees. This interpretation is somewhat speculative because we have no direct evidence for their association, but warrants further investigation.

In summary, the collective findings of this study reveal that non-genetic factors, and to a lesser extent, genetic factors appear to influence mutual eye gaze in adult chimpanzees. Additionally, individual variation in mutual eye gaze bout length is associated with gray matter covariation in the visual cortex, inferior and middle temporal cortex, and the basal forebrain. These findings are the first systematic report of neuroanatomical correlates of individual variation in chimpanzee mutual eye gaze; however, more studies are needed to further identify the neurogenomic mechanisms and consequences that underlie variation in mutual eye gaze. Continued studies in chimpanzees and other primates will further advance our understanding of the neurochemical, psychological, evolutionary, and potential clinical relevance of mutual eye gaze.

\section{Methods}

Subjects. For the behavioral studies, there were 219 adult chimpanzees (Pan troglodytes; $11-59$ years old; 138 females, 81 males) from two facilities: the Yerkes National Primate Research Center (YNPRC, N =69) and the National Center for Chimpanzee Care (NCCC, $\mathrm{N}=150$ ) of The University of Texas MD Anderson Cancer Center. All chimpanzees were socially housed with 24-hour access to indoor/outdoor enclosures (except during cleaning) with bedding, climbing structures, and daily environmental enrichment. Chimpanzees were fed a commercially available primate diet and fresh produce, with daily foraging opportunities and ad libitum access to water. 
Of the 219 subjects, we included 125 mother-reared, 52 nursery-reared, and 42 wild-born apes. As we have previously defined ${ }^{12,60}$, nursery-reared chimpanzees were separated from their mother (between 0-30 days old) due to unresponsive care, injury, or illness. These infants were hand-reared and fed human infant formula (not supplemented with DHA as far as we know) until they could sufficiently care for themselves. They were placed with same-age peers until three years old when they were integrated into larger mixed-age social groups (including adults and sub-adults) $)^{12,60}$. Mother-reared chimpanzees were reared by their mother in their natal groups (4-20 chimpanzees) for at least the first 2.5 years of life. Wild-born apes were brought to the United States prior to the 1974 CITES importation ban. Of the 219 chimpanzees for which behavioral data were available, we also had structural brain scans available for 191 individuals (79 males, 112 female; 104 mother-reared, 54 nursery-reared, and 33 wild-born). All work was approved by the Institutional Animal Care and Use Committees at both NCCC and YNPRC and adhered to the ethical guidelines of National Institutes of Health.

Mutual eye gaze. A familiar experimenter (over 8 years of experience with the chimpanzees) tested each individual during four 1-minute test trials that were separated by at least one day. Each trial began with the experimenter greeting the subject and giving the subject (and any present group members) a small piece of food (i.e., a peanut or grape). The experimenter then placed the food out of sight of the apes, and sat or stood (depending on the subject's position) approximately 2-3 feet from the subject's home cage (close enough to feel confident knowing when eye contact was achieved). A trial did not begin until the subject appeared settled and focused on the experimenter with outside activity and noise at a minimum. To initiate a trial, the experimenter made direct eye contact with the subject. For the subsequent 60 seconds, the total amount of time and frequency of mutual eye gaze events were recorded with a stopwatch by the experimenter. Throughout the 1-minute trial, the experimenter actively sought to maintain eye contact with the subject, including calling its name, if for some reason it turned away from the experimenter. Though rare in occurrence, trials were terminated if the subject left the area and went out of view from the experimenter, or if an event (i.e., alarm barking, fighting in the group) caused the subject's attention to be significantly diverted. For example, chimpanzees at NCCC were only distracted and required re-testing for 6 trials. Subjects were tested in their social groups and no attempt was made to isolate them for testing. For each trial, the number of mutual eye gaze events and the total duration in mutual eye gaze were recorded and served as dependent measures in subsequent analyses. Additionally, average bout length of mutual eye gaze was computed for each trial by dividing the total duration of MEG by the frequency of MEG events.

MRI acquisition and post-image processing. We used magnetic resonance image (MRI) scans that were acquired during one of the chimpanzee's past annual physical examinations (as previously reported ${ }^{25,61-63}$ ). Briefly, the animals were first sedated, then subsequently anesthetized, before being transported to a mobile MRI facility. For safety purposes, chimpanzees were briefly housed alone for 2-24hours while recovering from anesthesia before being reunited with their social group. All procedures were approved by the Institutional Animal Care and Use Committees at YNPRC and NCCC, and also followed the guidelines of the Institute of Medicine on the use of chimpanzees in research. For this study, 77 YNPRC chimpanzees were scanned using a 3.0 Tesla scanner (Siemens Trio, Siemens Medical Solutions USA, Inc., Malvern, Pennsylvania, USA), while the remaining 149 chimpanzees (11 from YNPRC, 138 from NCCC) were scanned using a 1.5T GE echo-speed Horizon LX MR scanner (GE Medical Systems, Milwaukee, Wisconsin, USA).

Following acquisition, we used a Macintosh computer to process all of the MRI scans using recently published procedures ${ }^{63}$. We completed all processing twice (for the 1.5T and 3T scans separately). Briefly, we converted raw DICOM files into NiFTI format using 3D Slicer 4 (www.3Dslicer.org) ${ }^{64,65}$, then used the brain extraction function in FSL for skull stripping ${ }^{66,67}$. Subsequently, we bias corrected and denoised each image using 3D Slicer ${ }^{68-71}$ and the DWI Denoising Package for MATLAB (R2015b; Mathworks, Natick, Massachusetts, USA) ${ }^{72,73}$. We then resampled the scans (at $0.625 \mathrm{~mm}$ ), aligned each on the AC-PC axis, and saved them in radiological space (all subjects had a directional marker placed before imaging) using ANALYZE 11.0 (AnalyzeDirect, Overland Park, Kansas, USA). Finally, we registered each scan to a chimpanzee template brain ${ }^{61}$ using a 12-parameter affine linear registration in $\mathrm{FSL}^{74,75}$.

The AC-PC-aligned and affine-registered brains were then processed through the FSL-VBM pipeline (http:// fsl.fmrib.ox.ac.uk/fsl/fslwiki/FSLVBM) and subsequently reregistered to the chimpanzee template brain, using a 6-parameter rigid body linear registration in FSL (FLIRT) ${ }^{61,74,75}$, ensuring that the 1.5T and 3T scans had the same orientation and voxel dimensions (see detailed processing methods in $^{63}$ ).

SBM analysis. The SBM analysis was performed with the software program available in the toolbox GIFT (http://icatb.sourceforge.net) on the smoothed, modulated gray matter volumes for the 191 chimpanzees for which MRI and MEG data were available. The individual chimpanzee's smoothed gray matter volumes were imported into the program and subjected to an independent component analysis using the default parameters. For this set of MRI scans, the SBM produced 18 gray matter components. SBM then computes a weighted score for each subject that reflects their contribution to the creation of each gray matter covariation component. These weighted scores for the 18 components were the outcome measures of interest for subsequent phenotypic and heritability analyses.

Heritability analyses. We used the software program SOLAR ${ }^{76}$ to determine the heritability of each MEG measure ( $\mathrm{se}^{25}$ for detailed methods). Researchers have used SOLAR extensively with nonhuman primates for a

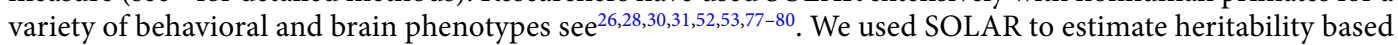
on the chimpanzee pedigree, and to determine whether the three MEG measures were significantly heritable. We then used SOLAR to run genetic correlations (RhoG) to determine the extent to which common genetic 
mechanisms explained both the MEG measures and the correlated SBM component scores. We included sex, age, rearing history, and scanner magnet (when appropriate) as covariates for all genetic analyses.

\section{Data availability}

All MRI scans and datasets analyzed in the current study will be available through the National Chimpanzee Brain Resource data repository (www.chimpanzeebrain.org).

Received: 11 December 2019; Accepted: 9 April 2020;

Published online: 04 May 2020

\section{References}

1. Grossmann, T. The eyes as windows into other minds: An integrative perspective. Perspectives on. Psychological Science 12, 107-121, https://doi.org/10.1177/1745691616654457 (2017).

2. Simpson, E. A., Paukner, A., Pedersen, E. J., Ferrari, P. F. \& Parr, L. A. Visual preferences for direct-gaze faces in infant macaques (Macaca mulatta) with limited face exposure. Developmental Psychobiology 61, 228-238 (2019).

3. Flom, R., Lee, K. S. \& Muir, D. (Psychology Press, London, 2006).

4. Kristen-Antonow, S., Sodian, B., Perst, H. \& Licata, M. A longitudinal study of the emerging self from 9 months to the age of 4 years. Frontiers in Psychology 6, 789, https://doi.org/10.3389/fpsyg.2015.00789 (2015).

5. Kano, F. \& Call, J. Cross-species variation in gaze following and conspecific preference among great apes, human infants and adults. Animal Behaviour 91, 137-150 (2014).

6. Farran, D. C. \& Kasari, C. A longitudinal analysis of the development of synchrony in mutual gaze in mother-child dyads. Journal of Applied Developmental Psychology 11, 419-430, https://doi.org/10.1016/0193-3973(90)90018-f (1990).

7. Ferrari, P. F., Paukner, A., Ionica, C. \& Suomi, S. J. Reciprocal face-to-face communication between rhesus macaque mothers and their newborn infants. Current Biology 19, 1768-1772, https://doi.org/10.1016/j.cub.2009.08.055 (2009).

8. Niedzwiecka, A., Ramotowska, S. \& Tomalski, P. Mutual gaze during early mother-infant interactions promotes attention control development. Child Development 89, 2230-2244, https://doi.org/10.1111/cdev.12830 (2018).

9. Emery, N. J. The eyes have it: The neuroethology, function and evolution of social gaze. Neuroscience and Biobehavioral Reviews 24, 581-604, https://doi.org/10.1016/S0149-7634(00)00025-7 (2000).

10. Osterling, J. A., Dawson, G. \& Munson, J. A. Early recognition of 1-year-old infants with autism spectrum disorder versus mental retardation. Development and Psychopathology 14, 239-251 (2002).

11. Jones, W., Carr, K. \& Klin, A. Absence of preferential looking to the eyes of approaching adults predicts level of social disability in 2-year-old toddlers with autism spectrum disorder. Arch Gen Psychiatry 65, 946-954, https://doi.org/10.1001/archpsyc.65.8.946 (2008).

12. Bard, K. A. Evolutionary roots of intuitive parenting: Maternal competence in chimpanzees. Early Development and Parenting 3 , 19-28, https://doi.org/10.1002/edp.2430030104 (1994).

13. Brauer, J., Call, J. \& Tomasello, M. All great ape species follow gaze to distant locations and around barriers. J Comp Psychol 119, 145-154 (2005).

14. Tomasello, M., Call, J. \& Hare, B. Five primate species follow the visual gaze of conspecifics. Animal Behaviour 55, 1063-1069 (1998).

15. Tomasello, M., Hare, B. \& Agnetta, B. Chimpanzees, Pan troglodytes, follow gaze direction geometrically. Animal Behaviour 58, 769-777, https://doi.org/10.1006/anbe.1999.1192 (1999).

16. Tomasello, M., Hare, B., Lehmann, H. \& Call, J. Reliance on head versus eyes in the gaze following of great apes and human infants: The cooperative eye hypothesis. Journal of Human Evolution 52, 314-320, https://doi.org/10.1016/j.jhevol.2006.10.001 (2007).

17. Amici, F., Aureli, F., Visalberghi, E. \& Call, J. Spider monkeys (Ateles geoffroyi) and capuchin monkeys (Cebus apella) follow gaze around barriers: Evidence for perspective taking? Journal of Comparative Psychology 123, 368-374 (2009).

18. Rosati, A. G. \& Hare, B. Looking past the model species: Diversity in gaze-following skills in primates. Current Opinion in Neurobiology 19, 45-51 (2009).

19. Kano, F., Hirata, S. \& Call, J. Social attention in the two species of Pan: Bonobos make more eye contact than chimpanzees. PLoS One 15, e0129684 (2015).

20. Bard, K. A. et al. Group differences in the mutual gaze of chimpanzees (Pan troglodytes). Developmental Psychology 41, 616-624, https://doi.org/10.1037/0012-1649.41.4.616 (2005).

21. Itier, R. J. \& Batty, M. Neural bases of eye and gaze processing: The core of social cognition. Neuroscience and Biobehavioral Reviews 33, 843-863 (2009).

22. Kamphius, S., Dicke, P. W. \& Thier, P. Neuronal substrates for gaze following in monkeys. European Journal of Neuroscience 29, 1732-1738 (2009).

23. Shepherd, S. Following gaze: Gaze-following behavior as a window to social cognition. Frontiers in Integrative. Neuroscience 4, 1 (2010).

24. Roy, A., Shepard, S. V. \& Platt, M. L. Reversible inactivation of pSTS supresses social gaze following in the macaque (Macaca mulatta). Social Cognitive and Affective Neuroscience 9, 209-217 (2012).

25. Hopkins, W. D. et al. Heritability of Gray Matter Structural Covariation and Tool Use Skills in Chimpanzees (Pan troglodytes): A Source-Based Morphometry and Quantitative Genetic Analysis. Cerebral Cortex 29, 3702-3711 (2018).

26. Hopkins, W. D., Mareno, M. C. \& Schapiro, S. J. Further evidence of a left hemisphere specialization and genetic basis for tool use skill in chimpanzees (Pan troglodytes): Reproducibility in two genetically isolated populations of apes. Journal of Comparative Psychology 133, 512-519 (2019).

27. Phillips, K. A., Rogers, J., Barrett, E. A., Glahn, D. C. \& Kochunov, P. V. Genetic contributions to the midsagittal area of the corpus callosum. Twin Research and Human Genetics 15, 315-323 (2012).

28. Rogers, J. et al. Heritability of brain volume, surface area and shape: An MRI study in an extended pedigree of baboons. Human Brain Mapping 28, 576-583 (2007).

29. Rogers, J. et al. On the genetic architecture of cortical folding and brain volume in primates. NeuroImage 53, 1103-1108 (2010).

30. Fairbanks, L. A. et al. Environmental stress alters genetic regulation of novelty seeking in vervet monkeys. Genes, Brain and Behavior 10, 683-688 (2011).

31. Fears, S. C. et al. Identifying heritable brain phenotypes in an extended pedigree of vervet monkeys. The Journal of Neuroscience 29, 2867-2875 (2009).

32. Hopkins, W. D. et al. Genetic influences on receptive joint attention in chimpanzees (Pan troglodytes). Scientific Reports 4, 1-7 (2014).

33. Russell, J. L., Lyn, H., Schaeffer, J. A. \& Hopkins, W. D. The role of socio-communicative rearing environments in the development of social and physical cognition in apes. Developmental Science 14, 1459-1470 (2011).

34. Lyn, H., Russell, J. L. \& Hopkins, W. D. The impact of environment on the comprehension of declarative communication in apes. Psychological Science 21, 360-365 (2010).

35. Itakura, S., Agnetta, B., Hare, B. \& Tomasello, M. Chimpanzee use of human and conspecific social cues to locate hidden food. Developmental Science 2, 448-456 (1999). 
36. Call, J. \& Tomasello, M. Production and comprehension of referential pointing by orangutans (Pongo pygmaeus). Journal of Comparative Psychology 108, 307-317 (1994).

37. Call, J. \& Tomasello, M. In Reaching into thought:The minds of the great apes (ed A. E. Russon, Bard, K. A., \& Parker, S. T.) 371-403 (Cambridge University Press, 1996).

38. Xu, L., Groth, K. M., Pearlson, G., Schreffen, D. J. \& Calhoun, V. D. Source-based morphometry: The use of independent component analysis to identify gray matter differences with application to schizophrenia. Human Brain Mapping 30, 711-724 (2009).

39. Senju, A. et al. Early social experience affects the development of eye gaze processing. Current Biology 25, 3086-3091, https://doi. org/10.1016/j.cub.2015.10.019 (2015).

40. Leavens, D. A. \& Hopkins, W. D. Intentional communication by chimpanzee (Pan troglodytes): A cross-sectional study of the use of referential gestures. Developmental Psychology 34, 813-822 (1998).

41. Seres, M., Autreli, F. \& de Waal, F. B. Successful formation of a large chimpanzees group out of two existing groups. Zoo Biology 20, 501-515 (2001)

42. Thunstrom, M., Persson, T. \& Bjorklund, M. Integration of a hand-reared chimpanzee (Pan troglodytes) infant into a social group of conspecifics. Primates 54, 13-19, https://doi.org/10.1007/s10329-012-0319-1 (2013).

43. Bashaw, M. J., Gullott, R. L. \& Gill, E. C. What defines successful integration into a social group for hand-reared chimpanzee infants? Primates 51, 139-147, https://doi.org/10.1007/s10329-009-0176-8 (2010).

44. Hall, K. et al. Chimpanzee uses manipulative gaze cues to conceal and reveal information to foraging competitor. American journal of primatology 79, e22622 (2017).

45. Dawson, G. et al. Neurocognitive and electrophysiological evidence of altered face processing in parents of children with autism: Implications for a model of abnormal development of social brain circuitry in autism. Development and Psychopathology 17, 679-697, https://doi.org/10.1017/S0954579405050327 (2005).

46. Frazier, T. W. et al. A meta-analysis of gaze differences to social and nonsocial information between individuals with and without autism. Journal of the American Academy of Child \& Adolescent Psychiatry 56, 546-555, https://doi.org/10.1016/j.jaac.2017.05.005 (2017).

47. Jones, E. J. et al. Reduced engagement with social stimuli in 6-month-old infants with later autism spectrum disorder: A longitudinal prospective study of infants at high familial risk. Journal of Neurodevelopmental Disorders 8, 7 (2016).

48. Rossion, B. \& Taubert, J. What can we learn about human individual face recognition from experimental studies in monkeys? Vision Research 157, 142-158, https://doi.org/10.1016/j.visres.2018.03.012 (2019).

49. Speer, L. L., Cook, A. E., McMahon, W. M. \& Clark, E. Face processing in children with autism: Effects of stimulus contents and type. Autism 11, 265-277 (2007).

50. Kano, F., Shepherd, S. V., Hirata, S. \& Call, J. Primate social attention: Species differences and effects of individual experience in humans, great apes, and macaques. PloS one 13, e0193283 (2018).

51. Fagot, J. Picture perception in animals. (Psychology Press, 2000).

52. Hopkins, W. D. et al. Poor receptive joint attention skills are associated with atypical grey matter asymmetry in the posterior superior temporal gyrus of chimpanzees (Pan troglodytes). Frontiers in Cognition 5, 1-8 (2014).

53. Hopkins, W. D., Russell, J. L. \& Schaeffer, J. Chimpanzee intelligence is heritable. Current Biology 24, 1649-1652, https://doi. org/10.1016/j.cub.2014.05.076 (2014)

54. Bard, K. A. \& Hopkins, W. D. Early socioemotional intervention mediates long-term effects of atypical rearing on structural covariation in gray matter in adult chimpanzees. Psychological Science 29, 594-603, https://doi.org/10.1177/0956797617740685 (2018).

55. Ikemoto, S. \& Panksepp, J. The role of nucleus accumbens dopamine in motivated behavior: A unifying interpretation with special reference to reward-seeking. Brain Research Reviews 31, 6-41 (1999).

56. Baskerville, T. A. \& Douglas, A. J. Dopamine and oxytocin interactions underlying behaviors: potential contributions to behavioral disorders. CNS Neuroscience \& Therapeutics 16, e92-123, https://doi.org/10.1111/j.1755-5949.2010.00154.x (2010).

57. Love, T. M. Oxytocin, motivation and the role of dopamine. Pharmacology Biochemistry and Behavior 119, 49-60, https://doi. org/10.1016/j.pbb.2013.06.011 (2014).

58. Young, L. J. \& Alexander, B. The chemistry between us: Love, sex and the science of attraction. (Penguin Group, 2014).

59. Nagasawa, M. et al. Social evolution. Oxytocin-gaze positive loop and the coevolution of human-dog bonds. Science 348, 333-336, https://doi.org/10.1126/science.1261022 (2015).

60. Bard, K. A., Platzman, K. A., Lester, B. M. \& Suomi, S. J. Orientation to social and nonsocial stimuli in neonatal chimpanzees and humans. Infant Behavior and Development 15, 43-56, https://doi.org/10.1016/0163-6383(92)90005-q (1992).

61. Hopkins, W. D. \& Avants, B. B. Regional and hemispheric variation in cortical thickness in chimpanzees (Pan troglodytes). Journal of Neuroscience 33, 5241-5248 (2013).

62. Hopkins, W. D. et al. Motor skill for tool-use is associated with asymmetries in Broca's area and the motor hand area of the precentral gyrus in chimpanzees (Pan troglodytes). Behavioural Brain Research 318, 71-81 (2017).

63. Mulholland, M. M. et al. AVPR1A variation is linked to gray matter covariation in the social brain network of chimpanzees. Genes, Brain and Behavior 19, e12631, https://doi.org/10.1111/gbb.12631 (2020).

64. Kikinis, R., Pieper, S. D. \& Vosburgh, K. G. In Intraoperative imaging and image-guided therapy 277-289 (Springer Science \& Business Media, 2014).

65. Fedorov, A. et al. 3D Slicer as an image computing platform for the Quantitative Imaging Network. Magnetic resonance imaging 30, 1323-1341 (2012).

66. Smith, S. M. Fast robust automated brain extraction. Human Brain Mapping 17, 143-155, https://doi.org/10.1002/hbm.10062 (2002).

67. Jenkinson, M., Pechaud, M. \& Smith, S. BET2: MR-based estimation of brain, skull and scalp surfaces. Eleventh Annual Meeting of the Organization for Human Brain Mapping 17, 167 (2005).

68. Tustison, N. \& Gee, J. N4ITK: Nick's N3 ITK implementation for MRI bias field correction. Insight Journal 9 (2009).

69. Tustison, N., Avants, B. B., Cook, P. A., \& Gee, J. C. (2010). N4ITK: Improved n3 bias correction with robust b-spline approximation. Paper presented at the Proc. of ISBI. (2010).

70. Boyes, R. G. et al. Intensity non-uniformity correction using N3 on 3-T scanners with multichannel phased array coils. Neuroimage 39, 1752-1762 (2008).

71. Tustison, N. J. et al. Large-scale evaluation of ANTs and FreeSurfer cortical thickness measurements. Neuroimage 99, 166-179 (2014).

72. Coupé, P. \& Manjon, J. MRI Denoising Software for Matlab [Computer software]. (2013)

73. Coupé, P. et al. An optimized blockwise nonlocal means denoising filter for 3-D magnetic resonance images. IEEE Transactions on Medical Imaging 27, 425-441 (2008).

74. Jenkinson, M. \& Smith, S. A global optimisation method for robust affine registration of brain images. Medical Image Analysis 5, $143-156(2001)$

75. Jenkinson, M., Bannister, P., Brady, M. \& Smith, S. Improved optimization for the robust and accurate linear registration and motion correction of brain images. Neuroimage 17, 825-841 (2002).

76. Almasy, L. \& Blangero, J. Multipoint quantitative-trait linkage analysis in general pedigrees. American Journal of Human Genetics 62, 1198-1211 (1998). 
77. Fears, S. C. et al. Anatomic brain asymmetry in vervet monkeys. PlosOne 6, e28243 (2011).

78. Hopkins, W. D., Stimpson, C. D. \& Sherwood, C. C. in Bonobos: Unique in Mind, Brain, and Behavior 199-213 (Oxford University Press, 2018).

79. Hopkins, W. D. et al. Genetic factors and oro-facial motor learning selectively influence variability in central sulcus morphology in chimpanzees (Pan troglodytes). Journal of Neuroscience 37, 5475-5483 (2017).

80. Johnson, Z. et al. Genetic influences on response to novel objects and dimensions of personality in Papio baboons. Behav Genet 45 , 215-227, https://doi.org/10.1007/s10519-014-9702-6 (2015).

\section{Acknowledgements}

This work was supported by the National Institutes of Health [grant numbers NS-42867, NS-73134, and HD60563], and the National Science Foundation [INSPIRE grant number 1542848]. The chimpanzees at the National Center for Chimpanzee Care (NCCC) are supported by the National Institutes of Health [Cooperative Agreement U42-OD011197]. The Yerkes National Primate Research Center and the NCCC are fully accredited by AAALAC International. All work was carried out in accordance with the National Institutes of Health guide for the care and use of Laboratory animals and was approved by each facility's Institutional Animal Care and Use Committee.

\section{Author contributions}

W.D.H. was responsible for the conception, design, supervision, data acquisition, data analysis and interpretation, and wrote the main manuscript text. M.M.M. was responsible for data processing and analysis, and wrote the main manuscript text. L.A.R. was responsible for acquisition of the data and revision of the manuscript text. M.C.M. was responsible for acquisition of the data and revision of the manuscript text. S.J.S. was responsible for supervision, data acquisition, and revision of the manuscript text. All authors approved the submitted version and are accountable for the work.

\section{Competing interests}

The authors declare no competing interests.

\section{Additional information}

Correspondence and requests for materials should be addressed to W.D.H. or M.M.M.

Reprints and permissions information is available at www.nature.com/reprints.

Publisher's note Springer Nature remains neutral with regard to jurisdictional claims in published maps and institutional affiliations.

(c) (i) Open Access This article is licensed under a Creative Commons Attribution 4.0 International License, which permits use, sharing, adaptation, distribution and reproduction in any medium or format, as long as you give appropriate credit to the original author(s) and the source, provide a link to the Creative Commons license, and indicate if changes were made. The images or other third party material in this article are included in the article's Creative Commons license, unless indicated otherwise in a credit line to the material. If material is not included in the article's Creative Commons license and your intended use is not permitted by statutory regulation or exceeds the permitted use, you will need to obtain permission directly from the copyright holder. To view a copy of this license, visit http://creativecommons.org/licenses/by/4.0/.

(C) The Author(s) 2020 